MACIEJ WiLcZYŃSKI (Wrocław)

\title{
MINIMAX NONPARAMETRIC PREDICTION
}

Abstract. Let $U_{0}$ be a random vector taking its values in a measurable space and having an unknown distribution $P$ and let $U_{1}, \ldots, U_{n}$ and $V_{1}, \ldots, V_{m}$ be independent, simple random samples from $P$ of size $n$ and $m$, respectively. Further, let $z_{1}, \ldots, z_{k}$ be real-valued functions defined on the same space. Assuming that only the first sample is observed, we find a minimax predictor $\boldsymbol{d}^{0}\left(n, U_{1}, \ldots, U_{n}\right)$ of the vector $\boldsymbol{Y}^{m}=\sum_{j=1}^{m}\left(z_{1}\left(V_{j}\right), \ldots\right.$ $\left.\ldots, z_{k}\left(V_{j}\right)\right)^{T}$ with respect to a quadratic errors loss function.

1. Introduction. Let $U_{0}$ be a random vector taking its values in a measurable space $(\mathcal{Y}, \mathcal{B})$ and having an unknown distribution $P$, which is assumed to be an element of the set

$$
\mathcal{P}=\{\text { all probability measures on }(\mathcal{Y}, \mathcal{B})\} .
$$

Let $U_{1}, \ldots, U_{n}$ and $V_{1}, \ldots, V_{m}$ be independent, simple random samples from $P$ of size $n$ and $m$, respectively. Further, let $\boldsymbol{z}=\left(z_{1}, \ldots, z_{k}\right)^{T}$ be a measurable function on the space $(\mathcal{Y}, \mathcal{B})$ with values in $\left(\mathbb{R}^{k}, \mathcal{B}_{\mathbb{R}^{k}}\right)$. In the paper we consider the problem of predicting the value of a $k$-dimensional random vector $\boldsymbol{Y}^{m}=\sum_{j=1}^{m} \boldsymbol{z}\left(V_{j}\right)$ from the data $\boldsymbol{U}^{n}=\left(U_{1}, \ldots, U_{n}\right)$. Assuming that the loss function has the form

$$
L\left(\boldsymbol{d}, \boldsymbol{Y}^{m}\right)=\left(\boldsymbol{d}-\boldsymbol{Y}^{m}\right)^{T} \boldsymbol{C}\left(\boldsymbol{d}-\boldsymbol{Y}^{m}\right),
$$

where $C=\left[c_{i j}\right]$ is a nonnegative definite, symmetric $k \times k$ matrix, we find a minimax solution of the above prediction problem. We show that the minimax predictor $\boldsymbol{d}^{0}\left(n, \boldsymbol{U}^{n}\right)$ of $\boldsymbol{Y}^{m}$ is an affine (inhomogeneous linear) function of the random vector $\boldsymbol{X}^{n}=\sum_{j=1}^{n} \boldsymbol{z}\left(U_{j}\right)$.

The decision rule $\boldsymbol{d}^{0}\left(n, \boldsymbol{U}^{n}\right)$ has a risk function which is not constant and therefore proving its minimaxity cannot be accomplished by showing

2000 Mathematics Subject Classification: 62C20, 62 G05.

Key words and phrases: minimax prediction, Dirichlet process, nonparametric prediction. 
that this predictor is Bayes with respect to some prior on $\mathcal{P}$. Instead, we use the method proposed in Wilczyński (1992). First we show that $\boldsymbol{d}^{0}\left(n, \boldsymbol{U}^{n}\right)$ is minimax among all predictors which are affine functions of $\boldsymbol{X}^{n}$. Next, using some implications of this fact, we calculate the upper bound for a minimax risk of $\boldsymbol{d}^{0}\left(n, \boldsymbol{U}^{n}\right)$. Then, via nonparametric Bayes approach proposed by Ferguson (1973), we construct a sequence of priors on $\mathcal{P}$ for which the corresponding sequence of Bayes risks converges to this upper bound. From this we deduce minimaxity of $\boldsymbol{d}^{0}\left(n, \boldsymbol{U}^{n}\right)$.

2. Minimax estimate. The statement of our main result requires introducing the following notation. Let the function $\boldsymbol{z}_{*}:(\mathcal{Y}, \mathcal{B}) \rightarrow\left(\mathbb{R}^{k}, \mathcal{B}_{\mathbb{R}^{k}}\right)$ be defined by

$$
\boldsymbol{z}_{*}(y)=\boldsymbol{C}^{1 / 2} \boldsymbol{z}(y), \quad y \in Y,
$$

where $C^{1 / 2}$ is the square root of the matrix $C$, i.e. $C^{1 / 2} C^{1 / 2}=C$. The random vector $\boldsymbol{z}_{*}\left(U_{0}\right)$, its expected value and the sum of the variances of its components are denoted by $\boldsymbol{Z}_{*}, \boldsymbol{p}_{*}$ and $R_{1}(P)$ respectively, i.e. we put

$$
\begin{aligned}
\boldsymbol{Z}_{*} & =\boldsymbol{z}_{*}\left(U_{0}\right), \\
\boldsymbol{p}_{*} & =E_{P} \boldsymbol{Z}_{*}, \\
R_{1}(P) & =E_{P}\left\|\boldsymbol{Z}_{*}-E_{P} \boldsymbol{Z}_{*}\right\|^{2}=E_{P}\left\|\boldsymbol{Z}_{*}-\boldsymbol{p}_{*}\right\|^{2} .
\end{aligned}
$$

Now, let $\left(P_{j}\right)$ be any sequence of probability measures on $(\mathcal{Y}, \mathcal{B})$ such that

$$
\lim _{j \rightarrow \infty} R_{1}\left(P_{j}\right)=\sup _{P \in \mathcal{P}} R_{1}(P)
$$

and let $\left(\boldsymbol{b}_{j}\right)$ be the corresponding sequence of points from $\mathbb{R}^{k}$ defined by

$$
\boldsymbol{b}_{j}=E_{P_{j}} \boldsymbol{Z}_{*} \text {. }
$$

In Theorem 1, we show that the above prediction problem has a nontrivial solution only when the vector-valued function $\boldsymbol{z}_{*}(y)$ is bounded on $Y$, i.e. when

$$
M^{2}:=\sup _{y \in \mathcal{Y}}\left\|\boldsymbol{z}_{*}(y)\right\|^{2}=\sup _{y \in \mathcal{Y}} \boldsymbol{z}^{T}(y) \boldsymbol{C z}(y)<\infty .
$$

Obviously, if $M$ is finite then the random vector $\boldsymbol{Z}_{*}$ and its expected value $\boldsymbol{p}_{*}$ are bounded. This implies that $\sup _{P \in \mathcal{P}} R_{1}(P)<\infty$ and, because

$$
\left\|\boldsymbol{b}_{j}\right\|^{2}=\left\|E_{P_{j}} \boldsymbol{Z}_{*}\right\|^{2} \leq E_{P_{j}}\left\|\boldsymbol{Z}_{*}\right\|^{2} \leq M^{2}
$$

the sequence $\left(\boldsymbol{b}_{j}\right)$ takes its values in a convex compact subset $\mathcal{M}$ of $\mathbb{R}^{k}$, defined by

$$
\mathcal{M}=\left\{\boldsymbol{b} \in \mathbb{R}^{k}:\|\boldsymbol{b}\|^{2} \leq M^{2}\right\} .
$$

Therefore, this sequence has a cluster point $\boldsymbol{b}_{0} \in \mathcal{M}$. Now, we define $\boldsymbol{a}_{0}$ as a vector from $\mathbb{R}^{k}$ which solves the equation

$$
\boldsymbol{C}^{1 / 2} \boldsymbol{a}_{0}=\boldsymbol{b}_{0} .
$$


To see that $(5)$ can be solved, we denote by $\left(\boldsymbol{C}^{1 / 2}\right)^{-}$any g-inverse of the matrix $\boldsymbol{C}^{1 / 2}$. Since $\boldsymbol{C}^{1 / 2}\left(\boldsymbol{C}^{1 / 2}\right)^{-} \boldsymbol{C}^{1 / 2}=\boldsymbol{C}^{1 / 2}$, we have

$$
\begin{aligned}
\boldsymbol{b}_{j} & =E_{P_{j}} \boldsymbol{C}^{1 / 2} \boldsymbol{z}\left(U_{0}\right)=E_{P_{j}} \boldsymbol{C}^{1 / 2}\left(\boldsymbol{C}^{1 / 2}\right)^{-} \boldsymbol{C}^{1 / 2} \boldsymbol{z}\left(U_{0}\right) \\
& =\boldsymbol{C}^{1 / 2}\left(\boldsymbol{C}^{1 / 2}\right)^{-} E_{P_{j}} \boldsymbol{C}^{1 / 2} \boldsymbol{z}\left(U_{0}\right)=\boldsymbol{C}^{1 / 2}\left(\boldsymbol{C}^{1 / 2}\right)^{-} \boldsymbol{b}_{j} .
\end{aligned}
$$

This implies that $\left(\boldsymbol{C}^{1 / 2}\right)^{-} \boldsymbol{b}_{0}$ solves (5), because

$$
\boldsymbol{b}_{0}=\boldsymbol{C}^{1 / 2}\left(\boldsymbol{C}^{1 / 2}\right)^{-} \boldsymbol{b}_{0}=\boldsymbol{C}^{1 / 2} \boldsymbol{a}_{0}
$$

Now, let the number $\alpha_{0}$ satisfy the condition

$$
\alpha_{0}^{2} n+m=\left(\alpha_{0} n-m\right)^{2} \quad \text { and } \quad \alpha_{0} n-m<0
$$

i.e. let

$$
\alpha_{0}= \begin{cases}\frac{n m-\sqrt{n m(n+m-1)}}{n(n-1)} & \text { if } n>1, \\ \frac{m-1}{2} & \text { if } n=1 .\end{cases}
$$

The following theorem is the main result of the paper.

THEOREM 1. If $\sup _{y \in \mathcal{Y}} \boldsymbol{z}(y)^{T} \boldsymbol{C} \boldsymbol{z}(y)<\infty$ then

$$
\boldsymbol{d}^{0}\left(n, \boldsymbol{U}^{n}\right)=\alpha_{0} \boldsymbol{X}^{n}+\left(m-\alpha_{0} n\right) \boldsymbol{a}_{0}
$$

is a minimax predictor of the unobservable vector $\boldsymbol{Y}^{m}$ and its minimax risk equals

$$
\sup _{P \in \mathcal{P}} R\left(\boldsymbol{d}^{0}, P\right)=\left(\alpha_{0} n-m\right)^{2} \sup _{P \in \mathcal{P}} R_{1}(P) .
$$

If $\sup _{y \in \mathcal{Y}} \boldsymbol{z}(y)^{T} \boldsymbol{C} \boldsymbol{z}(y)=\infty$ then

$$
\inf _{d \in \mathcal{D}} \sup _{P \in \mathcal{P}} R(\boldsymbol{d}, P)=\infty
$$

and therefore a minimax predictor for $\boldsymbol{Y}^{m}$ does not exist.

3. Proof of the main result. Define the following two random vectors:

$$
\boldsymbol{X}_{*}^{n}=\boldsymbol{C}^{1 / 2} \boldsymbol{X}^{n}, \quad \boldsymbol{Y}_{*}^{n}=\boldsymbol{C}^{1 / 2} \boldsymbol{Y}^{n} .
$$

To prove the first part of Theorem 1 it suffices to show that the decision rule $\boldsymbol{d}_{*}^{0}\left(n, \boldsymbol{U}^{n}\right)=\boldsymbol{C}^{1 / 2} \boldsymbol{d}^{0}\left(n, \boldsymbol{U}^{n}\right)$, which, by (7) and (5), has the form

$$
\boldsymbol{d}_{*}^{0}\left(n, \boldsymbol{U}^{n}\right)=\boldsymbol{C}^{1 / 2} \boldsymbol{d}^{0}\left(n, \boldsymbol{U}^{n}\right)=\alpha_{0} \boldsymbol{X}_{*}^{n}+\left(m-\alpha_{0} n\right) \boldsymbol{b}_{0},
$$

is a minimax predictor of the vector $\boldsymbol{Y}_{*}^{m}$ under the loss function

$$
L_{*}\left(\boldsymbol{d}, \boldsymbol{Y}_{*}^{m}\right)=\left(\boldsymbol{d}-\boldsymbol{Y}_{*}^{m}\right)^{T}\left(\boldsymbol{d}-\boldsymbol{Y}_{*}^{m}\right)=\left\|\boldsymbol{d}-\boldsymbol{Y}_{*}^{m}\right\|^{2} .
$$

Moreover, to complete the proof of Theorem 1 it suffices to show that the risk function for any predictor of $\boldsymbol{Y}_{*}^{m}$ is unbounded when $\sup _{y \in \mathcal{Y}} \boldsymbol{z}(y)^{T} \boldsymbol{C} \boldsymbol{z}(y)$ $=\infty$. 
Let $\mathcal{D}$ be the class of all predictors $\boldsymbol{d}=\boldsymbol{d}\left(n, \boldsymbol{U}^{n}\right)$ of the vector $\boldsymbol{Y}_{*}^{m}$. We start the proof by calculating the risk function $R(\boldsymbol{d}, P)$ of any decision rule $\boldsymbol{d}$ from $\mathcal{D}$. Since the vectors $\boldsymbol{d}\left(n, \boldsymbol{U}^{n}\right)$ and $\boldsymbol{Y}_{*}^{m}=\sum_{j=1}^{m} \boldsymbol{z}_{*}\left(V_{j}\right)$ are independent, and since

$$
E_{P} \boldsymbol{Y}_{*}^{m}=\sum_{j=1}^{m} E_{P} \boldsymbol{z}_{*}\left(V_{j}\right)=m E_{P} \boldsymbol{z}_{*}\left(U_{0}\right)=m E_{P} \boldsymbol{Z}_{*}=m \boldsymbol{p}_{*},
$$

this risk is equal to

$$
R(\boldsymbol{d}, P)=E_{P}\left\|\boldsymbol{d}\left(n, \boldsymbol{U}^{n}\right)-\boldsymbol{Y}_{*}^{m}\right\|^{2}=E_{P}\left\|\boldsymbol{d}-m \boldsymbol{p}_{*}\right\|^{2}+E_{P}\left\|\boldsymbol{Y}_{*}^{m}-m \boldsymbol{p}_{*}\right\|^{2} .
$$

Moreover, since $\boldsymbol{z}_{*}\left(V_{1}\right), \ldots, \boldsymbol{z}_{*}\left(V_{m}\right)$ are i.i.d. random vectors with expected value $\boldsymbol{p}_{*}$,

$$
\begin{aligned}
E_{P}\left\|\boldsymbol{Y}_{*}^{m}-m \boldsymbol{p}_{*}\right\|^{2} & =E_{P}\left\|\sum_{j=1}^{m}\left(\boldsymbol{z}_{*}\left(V_{j}\right)-\boldsymbol{p}_{*}\right)\right\|^{2} \\
& =m E_{P}\left\|\boldsymbol{Z}_{*}-\boldsymbol{p}_{*}\right\|^{2}=m R_{1}(P) .
\end{aligned}
$$

Therefore, the risk for any predictor $\boldsymbol{d}\left(n, \boldsymbol{U}^{n}\right) \in \mathcal{D}$ may be rewritten as

$$
R(\boldsymbol{d}, P)=E_{P}\left\|\boldsymbol{d}-m \boldsymbol{p}_{*}\right\|^{2}+m R_{1}(P) .
$$

Assume now that $\sup _{y \in \mathcal{Y}} \boldsymbol{z}(y)^{T} \boldsymbol{C} \boldsymbol{z}(y)<\infty$. According to the definition of minimaxity, to prove that the predictor $\boldsymbol{d}_{*}^{0}\left(n, \boldsymbol{U}^{n}\right)$ defined by (8) is minimax we have to show that

$$
\sup _{P \in \mathcal{P}} R\left(\boldsymbol{d}_{*}^{0}, P\right)=\inf _{d \in \mathcal{D}} \sup _{P \in \mathcal{P}} R(\boldsymbol{d}, P) .
$$

To do this, we denote by $\mathcal{D}_{0}$ the following class of affine predictors:

$$
\mathcal{D}_{0}=\left\{\boldsymbol{d}^{\boldsymbol{b}} \in \mathcal{D}: \boldsymbol{d}^{\boldsymbol{b}}\left(n, \boldsymbol{U}^{n}\right)=\alpha_{0} \boldsymbol{X}_{*}^{n}+\left(m-\alpha_{0} n\right) \boldsymbol{b}, \boldsymbol{b} \in \mathcal{M}\right\},
$$

where the number $\alpha_{0}$ is defined by (6), and we prove that $\boldsymbol{d}_{*}^{0}=\boldsymbol{d}^{\boldsymbol{b}_{0}}$ is $\operatorname{minimax}$ in $\mathcal{D}_{0}$, i.e.

$$
\sup _{P \in \mathcal{P}} R\left(\boldsymbol{d}_{*}^{0}, P\right)=\inf _{d \in \mathcal{D}_{0}} \sup _{P \in \mathcal{P}} R(\boldsymbol{d}, P) .
$$

Next, using some implication of the minimaxity of $\boldsymbol{d}_{*}^{0}$ in $\mathcal{D}_{0}$, we calculate the upper bound for $R\left(\boldsymbol{d}_{*}^{0}, P\right)$. Then, if $m>1$, we construct a sequence of priors on $\mathcal{P}$ for which the corresponding sequence of Bayes risks converges to this upper bound. From this we deduce minimaxity of $\boldsymbol{d}_{*}^{0} \in \mathcal{D}$. If $m=1$, we use a different approach to prove that $\boldsymbol{d}_{*}^{0}$ is minimax in $\mathcal{D}$.

We start proving minimaxity of $\boldsymbol{d}_{*}^{0}$ in $\mathcal{D}_{0}$ by calculating its risk function. We first note that (cf. (9) and (10))

$$
\begin{aligned}
E_{P}\left\|\alpha_{0} \boldsymbol{X}_{*}^{n}+\left(m-\alpha_{0} n\right) \boldsymbol{b}-m \boldsymbol{p}_{*}\right\|^{2} & \\
& =\alpha_{0}^{2} E_{P}\left\|\boldsymbol{X}_{*}^{n}-n \boldsymbol{p}_{*}\right\|^{2}+\left(\alpha_{0} n-m\right)^{2}\left\|\boldsymbol{b}-\boldsymbol{p}_{*}\right\|^{2} \\
& =\alpha_{0}^{2} n R_{1}(P)+\left(\alpha_{0} n-m\right)^{2}\left\|\boldsymbol{b}-\boldsymbol{p}_{*}\right\|^{2} .
\end{aligned}
$$


Since $\alpha_{0}^{2} n+m=\left(\alpha_{0} n-m\right)^{2}$, we conclude, by (11), that the risk function for a predictor $\boldsymbol{d}^{\boldsymbol{b}} \in \mathcal{D}_{0}$, denoted for simplicity by $R(\boldsymbol{b}, P)$, is given by

$$
\begin{aligned}
R(\boldsymbol{b}, P) & =\left(\alpha_{0}^{2} n+m\right) R_{1}(P)+\left(\alpha_{0} n-m\right)^{2}\left\|\boldsymbol{b}-\boldsymbol{p}_{*}\right\|^{2} \\
& =\left(\alpha_{0} n-m\right)^{2}\left[R_{1}(P)+\left\|\boldsymbol{b}-\boldsymbol{p}_{*}\right\|^{2}\right] .
\end{aligned}
$$

Furthermore, if $\sup _{y \in \mathcal{Y}}\left\|\boldsymbol{z}_{*}(y)\right\|^{2}<\infty$ then the random vector $\boldsymbol{Z}_{*}$ and its expected value $\boldsymbol{p}_{*}=E_{P} \boldsymbol{Z}_{*}$ are bounded, and $R_{1}(P)$ can be rewritten as

$$
R_{1}(P)=E_{P}\left\|\boldsymbol{Z}_{*}-E_{P} \boldsymbol{Z}_{*}\right\|^{2}=E_{P}\left\|\boldsymbol{Z}_{*}\right\|^{2}-\left\|E_{P} \boldsymbol{Z}_{*}\right\|^{2} .
$$

Therefore,

$$
\begin{aligned}
R_{1}(P)+\left\|\boldsymbol{b}-\boldsymbol{p}_{*}\right\|^{2} & =E_{P}\left\|\boldsymbol{Z}_{*}-\boldsymbol{p}_{*}\right\|^{2}+\left\|\boldsymbol{b}-\boldsymbol{p}_{*}\right\|^{2} \\
& =E_{P}\left\|\boldsymbol{Z}_{*}\right\|^{2}-\left\|\boldsymbol{p}_{*}\right\|^{2}+\left\|\boldsymbol{b}-\boldsymbol{p}_{*}\right\|^{2} \\
& =E_{P}\left\|\boldsymbol{Z}_{*}\right\|^{2}-2 \boldsymbol{b}^{T} p_{*}+\|\boldsymbol{b}\|^{2} \\
& =E_{P}\left\|\boldsymbol{Z}_{*}\right\|^{2}-2 \boldsymbol{b}^{T} E_{P} \boldsymbol{Z}_{*}+\|\boldsymbol{b}\|^{2} .
\end{aligned}
$$

This implies that

$$
R(\boldsymbol{b}, P)=\left(\alpha_{0} n-m\right)^{2}\left[E_{P}\left\|\boldsymbol{Z}_{*}\right\|^{2}-2 \boldsymbol{b}^{T} E_{P} \boldsymbol{Z}_{*}+\|\boldsymbol{b}\|^{2}\right] .
$$

Obviously, to prove that the decision rule $\boldsymbol{d}_{*}^{0}\left(n, \boldsymbol{U}^{n}\right)$ defined by (8) is mini$\max$ in $\mathcal{D}_{0}$ it suffices to show that

$$
\sup _{P \in \mathcal{P}} R\left(\boldsymbol{b}_{0}, P\right)=\inf _{b \in \mathcal{M}} \sup _{P \in \mathcal{P}} R(\boldsymbol{b}, P) .
$$

For this we note that $\mathcal{M}$ and $\mathcal{P}$ are convex sets and $\mathcal{M}$ is compact. Moreover, for each fixed $P \in \mathcal{P}$, the mapping $R(\boldsymbol{b}, P): \mathcal{M} \times \mathcal{P} \rightarrow[0, \infty)$ is convex, continuous with respect to $\boldsymbol{b} \in \mathcal{M}$ and, for each fixed $\boldsymbol{b} \in \mathcal{M}$, it is, by (17), concave (linear) with respect $P \in \mathcal{P}$. This means that all the assumptions of the Nikaido theorem (see Aubin 1980, p. 217) are fulfilled and thus there exists a point $\underline{\boldsymbol{b}}$ for which

$$
\sup _{P \in \mathcal{P}} R(\underline{\boldsymbol{b}}, P)=\inf _{b \in \mathcal{M}} \sup _{P \in \mathcal{P}} R(\boldsymbol{b}, P)=\sup _{P \in \mathcal{P}} \inf _{b \in \mathcal{M}} R(\boldsymbol{b}, P) .
$$

Now it remains to prove that $\underline{\boldsymbol{b}}=\boldsymbol{b}_{0}$. We first observe that, by (19) and (15), the minimax risk in $\mathcal{D}_{0}$ equals

$$
\inf _{b \in \mathcal{M}} \sup _{P \in \mathcal{P}} R(\boldsymbol{b}, P)=\sup _{P \in \mathcal{P}} \inf _{b \in \mathcal{M}} R(\boldsymbol{b}, P)=\left(\alpha_{0} n-m\right)^{2} \sup _{P \in \mathcal{P}} R_{1}(P),
$$

because, for a fixed distribution $P \in \mathcal{P}$, the convex function $R(\boldsymbol{b}, P)$ of the variable $\boldsymbol{b}$ attains its global minimum over $\mathcal{M}$ at the point $\boldsymbol{b}(P)=\boldsymbol{p}_{*}$. Moreover, an easy computation shows that, for each $P \in \mathcal{P}, 0<\beta<1$ and $j \geq 1$,

$$
\begin{aligned}
\sup _{Q \in \mathcal{P}} R_{1}(Q) & \geq R_{1}\left(\beta P+(1-\beta) P_{j}\right) \\
& =\beta R_{1}(P)+(1-\beta) R_{1}\left(P_{j}\right)+\beta(1-\beta) \|\left(E_{P_{j}} \boldsymbol{Z}_{*}-E_{P} \boldsymbol{Z}_{*} \|^{2},\right.
\end{aligned}
$$


because $\beta P+(1-\beta) P_{j} \in \mathcal{P}$ and, by (16),

$$
\begin{aligned}
R_{1}(\beta P & \left.+(1-\beta) P_{j}\right)=E_{\beta P+(1-\beta) P_{j}}\left\|\boldsymbol{Z}_{*}\right\|^{2}-\left\|E_{\beta P+(1-\beta) P_{j}} \boldsymbol{Z}_{*}\right\|^{2} \\
= & \beta E_{P}\left\|\boldsymbol{Z}_{*}\right\|^{2}+(1-\beta) E_{P_{j}}\left\|\boldsymbol{Z}_{*}\right\|^{2}-\left\|\beta E_{P} \boldsymbol{Z}_{*}+(1-\beta) E_{P_{j}} \boldsymbol{Z}_{*}\right\|^{2} \\
= & \beta\left(E_{P}\left\|\boldsymbol{Z}_{*}\right\|^{2}-\left\|E_{P} \boldsymbol{Z}_{*}\right\|^{2}\right)+(1-\beta)\left(E_{P_{j}}\left\|\boldsymbol{Z}_{*}\right\|^{2}-\left\|E_{P_{j}} \boldsymbol{Z}_{*}\right\|^{2}\right) \\
& +\beta(1-\beta)\left\|E_{P_{j}} \boldsymbol{Z}_{*}-E_{P} \boldsymbol{Z}_{*}\right\|^{2} \\
= & \beta R_{1}(P)+(1-\beta) R_{1}\left(P_{j}\right)+\beta(1-\beta)\left\|E_{P_{j}} \boldsymbol{Z}_{*}-E_{P} \boldsymbol{Z}_{*}\right\|^{2} .
\end{aligned}
$$

Since $\boldsymbol{b}_{0}$ is a cluster point of the sequence $\left(\boldsymbol{b}_{j}\right)$, where $\boldsymbol{b}_{j}=E_{P_{j}} \boldsymbol{Z}_{*}$, and since $\lim _{j \rightarrow \infty} R_{1}\left(P_{j}\right)=\sup _{Q \in \mathcal{P}} R_{1}(Q)$, we conclude that

$$
\sup _{Q \in \mathcal{P}} R_{1}(Q) \geq \beta R_{1}(P)+(1-\beta) \sup _{Q \in \mathcal{P}} R_{1}(Q)+\beta(1-\beta)\left\|\boldsymbol{b}_{0}-E_{P} \boldsymbol{Z}_{*}\right\|^{2} .
$$

Therefore,

$$
\beta \sup _{Q \in \mathcal{P}} R_{1}(Q) \geq \beta R_{1}(P)+\beta(1-\beta)\left\|\boldsymbol{b}_{0}-E_{P} \boldsymbol{Z}_{*}\right\|^{2}
$$

and, since $\beta$ is positive,

$$
\sup _{Q \in \mathcal{P}} R_{1}(Q) \geq R_{1}(P)+(1-\beta)\left\|\boldsymbol{b}_{0}-E_{P} \boldsymbol{Z}_{*}\right\|^{2} .
$$

Letting $\beta \rightarrow 0^{+}$, we can see that

$$
\sup _{Q \in \mathcal{P}} R_{1}(Q) \geq R_{1}(P)+\left\|\boldsymbol{b}_{0}-E_{P} \boldsymbol{Z}_{*}\right\|^{2}=R_{1}(P)+\left\|\boldsymbol{b}_{0}-\boldsymbol{p}_{*}\right\|^{2},
$$

which implies, by (15), that

$$
\left(\alpha_{0} n-m\right)^{2} \sup _{Q \in \mathcal{P}} R_{1}(Q) \geq\left(\alpha_{0} n-m\right)^{2}\left[R_{1}(P)+\left\|\boldsymbol{b}_{0}-\boldsymbol{p}_{*}\right\|^{2}\right]=R\left(\boldsymbol{b}_{0}, P\right) .
$$

Because this is true for all $P \in \mathcal{P}$, it follows, by (20), that

$$
\sup _{P \in \mathcal{P}} R\left(\boldsymbol{b}_{0}, P\right) \leq\left(\alpha_{0} n-m\right)^{2} \sup _{P \in \mathcal{P}} R_{1}(P)=\inf _{b \in \mathcal{M}} \sup _{P \in \mathcal{P}} R(\boldsymbol{b}, P) \leq \sup _{P \in \mathcal{P}} R\left(\boldsymbol{b}_{0}, P\right) .
$$

Thus the predictor $\boldsymbol{d}_{*}^{0}\left(n, \boldsymbol{U}^{n}\right)=\boldsymbol{d}^{\boldsymbol{b}_{0}}\left(n, \boldsymbol{U}^{n}\right)$ is minimax in $\mathcal{D}_{0}$ and its minimax risk is given by

$$
\sup _{P \in \mathcal{P}} R\left(\boldsymbol{d}_{*}^{0}, P\right)=\left(\alpha_{0} n-m\right)^{2} \sup _{P \in \mathcal{P}} R_{1}(P) .
$$

To prove that $\boldsymbol{d}_{*}^{0}\left(n, \boldsymbol{U}^{n}\right)$ is minimax in $\mathcal{D}$ we assume first that $m=1$. Then $\alpha_{0}=0$ and, for any predictor $\boldsymbol{d} \in \mathcal{D}$, we obtain, by (11) and (21),

$$
\begin{aligned}
\sup _{P \in \mathcal{P}} R(\boldsymbol{d}, P) & \geq m \sup _{P \in \mathcal{P}} R_{1}(P)=\sup _{P \in \mathcal{P}} R_{1}(P)=\left(\alpha_{0} n-m\right)^{2} \sup _{P \in \mathcal{P}} R_{1}(P) \\
& =\sup _{P \in \mathcal{P}} R\left(\boldsymbol{d}_{*}^{0}, P\right),
\end{aligned}
$$

which implies minimaxity of $\boldsymbol{d}_{*}^{0}\left(n, \boldsymbol{U}^{n}\right)$ in the case where $m=1$. 
Now we assume that $m>1$. Then $\alpha_{0}>0$ and to show that $\boldsymbol{d}_{*}^{0}\left(n, \boldsymbol{U}^{n}\right)$ is minimax in $\mathcal{D}$ we make use of the nonparametric Bayes approach proposed in Ferguson (1973). The structure of the argument will be the same as in Wilczyński (1992).

For each $j \geq 1$ we denote by $\Pi_{j}$ a Dirichlet prior process on $(\mathcal{Y}, \mathcal{B})$ with parameter $\beta_{j}=\left[\left(m-\alpha_{0} n\right) / \alpha_{0}\right] P_{j}$, where $\left(P_{j}\right)$ is a sequence defined by $(3)$. From Ferguson (1973), Example b, the $\Pi_{j}$ Bayes nonparametric estimator of $m \boldsymbol{p}_{*}=m E_{P} \boldsymbol{Z}_{*}$, and therefore, by (11), the $\Pi_{j}$ Bayes nonparametric predictor of $\boldsymbol{Y}_{*}^{m}$ is given by

$$
\begin{aligned}
m\left[\frac{\left(m-\alpha_{0} n\right) / \alpha_{0}}{n+\left(m-\alpha_{0} n\right) / \alpha_{0}} E_{P_{j}} \boldsymbol{Z}_{*}+\frac{n}{n+\left(m-\alpha_{0} n\right) / \alpha_{0}} \cdot \frac{1}{n} \sum_{j=1}^{n} \boldsymbol{z}_{*}\left(U_{j}\right)\right] \\
=m\left[\frac{m-\alpha_{0} n}{m} \boldsymbol{b}_{j}+\frac{\alpha_{0}}{m} \boldsymbol{X}_{*}^{n}\right]=\boldsymbol{d}^{b_{j}}\left(n, \boldsymbol{U}^{n}\right) .
\end{aligned}
$$

Moreover, the Bayes risk $\varrho(j)$ for this decision rule has the form

$$
\begin{aligned}
\varrho(j) & :=E_{\Pi_{j}} R\left(\boldsymbol{b}_{j}, P\right)=\left(\alpha_{0} n-m\right)^{2}\left[E_{P_{j}}\left\|\boldsymbol{Z}_{*}\right\|^{2}-\left\|\boldsymbol{b}_{j}\right\|^{2}\right] \\
& =\left(\alpha_{0} n-m\right)^{2}\left[E_{P_{j}}\left\|\boldsymbol{Z}_{*}\right\|^{2}-\left\|E_{P_{j}} \boldsymbol{Z}_{*}\right\|^{2}\right]=\left(\alpha_{0} n-m\right)^{2} R_{1}\left(P_{j}\right),
\end{aligned}
$$

because, by (17),

$$
R\left(\boldsymbol{b}_{j}, P\right)=\left(\alpha_{0} n-m\right)^{2}\left[E_{P}\left\|\boldsymbol{Z}_{*}\right\|^{2}-2 \boldsymbol{b}_{j}^{T} E_{P} \boldsymbol{Z}_{*}+\left\|\boldsymbol{b}_{j}\right\|^{2}\right]
$$

and, by Ferguson (1973), Theorem 3,

$$
E_{\Pi_{j}}\left[E_{P}\left\|\boldsymbol{Z}_{*}\right\|^{2}\right]=E_{P_{j}}\left\|\boldsymbol{Z}_{*}\right\|^{2} \quad \text { and } \quad E_{\Pi_{j}}\left[E_{P} \boldsymbol{Z}_{*}\right]=E_{P_{j}} \boldsymbol{Z}_{*}=\boldsymbol{b}_{j} .
$$

As $j \rightarrow \infty$, the Bayes risk $\varrho(j)$ converges to $\left(\alpha_{0} n-m\right)^{2} \sup _{P \in \mathcal{P}} R_{1}(P)$, which, by (21), is the upper bound for the risk of $\boldsymbol{d}_{*}^{0}\left(n, U^{n}\right)$. This implies that $\boldsymbol{d}_{*}^{0}\left(n, \boldsymbol{U}^{n}\right)$ is a minimax predictor of $\boldsymbol{Y}_{*}^{m}$ (see Ferguson 1967, Theorem 2, p. 91) and thus the proof of the first part of Theorem 1 is complete.

We now turn to the proof of the second part. Since we assume that $\sup _{y \in \mathcal{Y}} \boldsymbol{z}(y)^{T} \boldsymbol{C} \boldsymbol{z}(y)=\infty$, there exists a sequence $\left(y_{j}\right) \subset \mathcal{Y}$ such that

$$
\lim _{j \rightarrow \infty}\left\|\boldsymbol{z}_{*}\left(y_{j}\right)\right\|^{2}=\infty .
$$

Let the distribution $P_{j}$ of $U_{0}$ be defined by

$$
P_{j}\left(U_{0}=y_{1}\right)=P_{j}\left(U_{0}=y_{j}\right)=0.5 \text {. }
$$

Then $\lim _{j \rightarrow \infty} R_{1}\left(P_{j}\right)=\sup _{P \in \mathcal{P}} R_{1}(P)=\infty$, because an easy calculation shows that

$$
R_{1}\left(P_{j}\right)=\frac{\left\|\boldsymbol{z}_{*}\left(y_{j}\right)-\boldsymbol{z}_{*}\left(y_{1}\right)\right\|^{2}}{4},
$$

and, by the triangle inequality and (23),

$$
\left\|\boldsymbol{z}_{*}\left(y_{j}\right)-\boldsymbol{z}_{*}\left(y_{1}\right)\right\| \geq\left\|\boldsymbol{z}_{*}\left(y_{j}\right)\right\|-\left\|\boldsymbol{z}_{*}\left(y_{1}\right)\right\| \rightarrow \infty .
$$


Therefore, the sequence of Bayes risks $\varrho(j)$ defined above converges to $\infty$. This implies, in turn, that the risk of any predictor $d\left(n, \boldsymbol{U}^{n}\right) \in \mathcal{D}$ is unbounded, because

$$
\sup _{P \in \mathcal{P}} R(\boldsymbol{d}, P) \geq E_{\Pi_{j}} R(\boldsymbol{d}, P) \geq \varrho(j) \rightarrow \infty .
$$

The proof of Theorem 1 is complete.

The first part of Theorem 1 can be slightly generalized. For this we denote by $\boldsymbol{I}$ the $k$-dimensional identity matrix and we put $H=\left(C^{1 / 2}\right)^{-} C^{1 / 2}$. Since $\boldsymbol{C}^{1 / 2}(\boldsymbol{I}-\boldsymbol{H})=\mathbf{0}$, we have the following result:

Corollary 1. If $\sup _{y \in \mathcal{Y}} \boldsymbol{z}(y)^{T} \boldsymbol{C z}(y)<\infty$ then, for each $\boldsymbol{c}_{0} \in \mathbb{R}^{k}$, the decision rule

$$
\boldsymbol{d}^{0}\left(n, \boldsymbol{U}^{n}\right)=\alpha_{0} \boldsymbol{X}^{n}+\left(m-\alpha_{0} n\right) \boldsymbol{a}_{0}+(\boldsymbol{I}-\boldsymbol{H}) c_{0}
$$

is a minimax predictor of $\boldsymbol{Y}^{m}$.

4. Examples. As an application of the results obtained we consider the following three examples.

ExAmple 1. Suppose that the set $\mathcal{Y}$ is centrosymmetric about $\mathbf{0}$ and that, for each $y \in \mathcal{Y}, \boldsymbol{z}_{*}(y)=-\boldsymbol{z}_{*}(-y)$. Let $\left(P_{j}\right)$ be a sequence for which (3) holds and let $P_{j}^{-}$denote the distribution of the random vector $-U_{0}$ whenever $U_{0}$ is distributed according to $P_{j}$. Then the sequence $\left(P_{j}^{\prime}\right)$, with $P_{j}^{\prime}=(1 / 2)\left(P_{j}+P_{j}^{-}\right)$, satisfies $(3)$, because

$$
\begin{aligned}
R_{1}\left(P_{j}^{\prime}\right) & =E_{P_{j}^{\prime}}\left\|\boldsymbol{Z}_{*}\right\|^{2}-\left\|E_{P_{j}^{\prime}} \boldsymbol{Z}_{*}\right\|^{2}=E_{P_{j}}\left\|\boldsymbol{Z}_{*}\right\|^{2}-\|\mathbf{0}\|^{2} \\
& \geq E_{P_{j}}\left\|\boldsymbol{Z}_{*}\right\|^{2}-\left\|E_{P_{j}} \boldsymbol{Z}_{*}\right\|^{2}=R_{1}\left(P_{j}\right) .
\end{aligned}
$$

Therefore, we may assume that $\boldsymbol{b}_{j}=E_{P_{j}^{\prime}} \boldsymbol{Z}_{*}=\mathbf{0}$, which implies that $\boldsymbol{b}_{0}=$ $\boldsymbol{a}_{0}=\mathbf{0}$ and thus the decision rule

$$
\boldsymbol{d}^{0}\left(n, \boldsymbol{U}^{n}\right)=\alpha_{0} \boldsymbol{X}^{n}
$$

is a minimax predictor of the unobservable vector $\boldsymbol{Y}^{m}$.

EXAmple 2. Suppose that $\boldsymbol{C}=\left[c_{i j}\right]$ is a diagonal matrix and that there exist two sequences $\left\{\bar{y}_{j}\right\}$ and $\left\{\overline{\bar{y}}_{j}\right\}$ in $\mathcal{Y}$ such that, for each $1 \leq i \leq k$,

$$
\lim _{j \rightarrow \infty} z_{i}\left(\bar{y}_{j}\right)=\inf _{y \in Y} z_{i}(y)>-\infty, \quad \lim _{j \rightarrow \infty} z_{i}\left(\overline{\bar{y}}_{j}\right)=\sup _{y \in Y} z_{i}(y)<\infty .
$$

Let the distribution $P_{j}$ of $U_{0}, j \geq 1$, be defined by

$$
P_{j}\left(U_{0}=\bar{y}_{j}\right)=P_{j}\left(U_{0}=\overline{\bar{y}}_{j}\right)=0.5 \text {. }
$$


Then it is easy to verify that for each $1 \leq i \leq k$,

$$
\begin{aligned}
\sup _{P \in \mathcal{P}}\left[E_{P}\left(z_{i}\left(U_{0}\right)\right)^{2}-\left(E_{P} z_{i}\left(U_{0}\right)\right)^{2}\right] & =\lim _{j \rightarrow \infty}\left[E_{P_{j}}\left(z_{i}\left(U_{0}\right)\right)^{2}-\left(E_{P_{j}} z_{i}\left(U_{0}\right)\right)^{2}\right] \\
& =\lim _{j \rightarrow \infty} \frac{\left|z_{i}\left(\bar{y}_{j}\right)-z_{i}\left(\overline{\bar{y}}_{j}\right)\right|^{2}}{4} .
\end{aligned}
$$

This implies that $\left(P_{j}\right)$ is a sequence of distributions as in (3), because $\boldsymbol{C}$ is assumed to be a diagonal matrix and thus

$$
R_{1}(P)=\sum_{i=1}^{k} c_{i i}\left[E_{P}\left(z_{i}\left(U_{0}\right)\right)^{2}-\left(E_{P} z_{i}\left(U_{0}\right)\right)^{2}\right] .
$$

Since the function $\boldsymbol{z}(y)$ is bounded on $\mathcal{Y}$,

$$
\begin{aligned}
\boldsymbol{C}^{1 / 2} a_{0} & =\boldsymbol{b}_{0}=\lim _{j \rightarrow \infty} E_{P_{j}} \boldsymbol{C}^{1 / 2} \boldsymbol{z}\left(U_{0}\right)=\boldsymbol{C}^{1 / 2} \lim _{j \rightarrow \infty} E_{P_{j}} \boldsymbol{z}\left(U_{0}\right) \\
& =\boldsymbol{C}^{1 / 2} \lim _{j \rightarrow \infty} \frac{\boldsymbol{z}\left(\bar{y}_{j}\right)+\boldsymbol{z}\left(\overline{\bar{y}}_{j}\right)}{2} .
\end{aligned}
$$

Therefore, the coordinates of the point $\boldsymbol{a}_{0}=\left(a_{01}, a_{02}, \ldots, a_{0 k}\right)^{T}$ are given by

$$
a_{0 i}=\lim _{j \rightarrow \infty} \frac{z_{i}\left(\bar{y}_{j}\right)+z_{i}\left(\overline{\bar{y}}_{j}\right)}{2}=\frac{\inf _{y \in \mathcal{Y}} z_{i}(y)+\sup _{y \in \mathcal{Y}} z_{i}(y)}{2}, \quad 1 \leq i \leq k,
$$

and

$$
\boldsymbol{d}^{0}\left(n, \boldsymbol{U}^{n}\right)=\alpha_{0} \boldsymbol{X}^{n}+\left(m-\alpha_{0} n\right) \boldsymbol{a}_{0}
$$

is a minimax predictor of the unobservable vector $\boldsymbol{Y}^{m}$.

EXAMPLE 3 . Let $\left\{A_{i}\right\}_{i=1}^{k}$ be a measurable partition of $\mathcal{Y}$, i.e. let $A_{1}, \ldots$ $\ldots, A_{k}$ be measurable, pairwise disjoint subsets of $\mathcal{Y}$ whose union equals $\mathcal{Y}$. Furthermore, let $z_{i}(y)=\mathbf{1}_{A_{i}}(y), 1 \leq i \leq k$, be the indicator functions. Then the random vectors $\boldsymbol{Z}=\boldsymbol{z}\left(U_{0}\right), \boldsymbol{X}^{n}$ and $\boldsymbol{Y}^{m}$ have $(1, \boldsymbol{p}),(n, \boldsymbol{p})$ and $(m, \boldsymbol{p})$ multinomial distributions, respectively, in which the parameter $\boldsymbol{p}=E_{P} \boldsymbol{Z}$ takes its values in the simplex $S$ defined by

$$
S=\left\{\left(s_{1}, \ldots, s_{k}\right): \text { for all } 1 \leq i \leq k, s_{i} \geq 0, \text { and } s_{1}+\ldots+s_{k}=1\right\} .
$$

Furthermore, it is easy to calculate that

$$
R_{1}(P)=\boldsymbol{c}^{T} \boldsymbol{p}-\boldsymbol{p}^{T} \boldsymbol{C} \boldsymbol{p},
$$

where $\boldsymbol{c}=\left(c_{11}, c_{22}, \ldots, c_{k k}\right)^{T}$ stands for the diagonal of the matrix $\boldsymbol{C}=\left[c_{i j}\right]$. This function attains its maximum over $\mathcal{P}$ at the distribution $P_{0}$ for which $E_{P_{0}} \boldsymbol{Z}=\boldsymbol{p}_{0}$, where

$$
\boldsymbol{c}^{T} \boldsymbol{p}_{0}-\boldsymbol{p}_{0}^{T} \boldsymbol{C} \boldsymbol{p}_{0}=\max _{p \in S}\left[\boldsymbol{c}^{T} \boldsymbol{p}-\boldsymbol{p}^{T} \boldsymbol{C p}\right]
$$


Therefore, $\boldsymbol{b}_{0}=E_{P_{0}} \boldsymbol{Z}_{*}=E_{P_{0}} \boldsymbol{C}^{1 / 2} \boldsymbol{Z}=\boldsymbol{C}^{1 / 2} E_{P_{0}} \boldsymbol{Z}=\boldsymbol{C}^{1 / 2} \boldsymbol{p}_{0}$ and $\boldsymbol{d}^{0}\left(n, \boldsymbol{U}^{n}\right)=\alpha_{0} \boldsymbol{X}^{n}+\left(m-\alpha_{0} n\right) \boldsymbol{p}_{0}$

is a minimax predictor of the unobservable vector $\boldsymbol{Y}^{m}$.

\section{References}

J. P. Aubin (1980), Mathematical Methods of Game and Economic Theory, North-Holland. T. S. Ferguson (1967), Mathematical Statistics: A Decision Theoretic Approach, Academic Press, New York.

T. S. Ferguson (1973), Bayesian analysis of some nonparametric problems, Ann. Statist. 1, 209-230.

M. Wilczyński (1992), Minimax nonparametric estimation, Sankhyā Ser. A 54, 425-431.

Institute of Mathematics

Wrocław University of Technology

Wybrzeże Wyspiańskiego 27

50-370 Wrocław, Poland

E-mail: wilczyn@neyman.im.pwr.wroc.pl

Received on 10.4.2000;

revised version on 27.9.2000 